\title{
Photon Recoil Momentum in Dispersive Media
}

\author{
Gretchen K. Campbell, Aaron E. Leanhardt*, Jongchul Mun, Micah \\ Boyd, Erik W. Streed, Wolfgang Ketterle and David E. Pritchard \\ MIT-Harvard Center for Ultracold Atoms, Research Laboratory of Electronics and Department of Physics, \\ Massachusetts Institute of Technology, Cambridge, MA 02139, USA
}

(Dated: February 2, 2008)

\begin{abstract}
A systematic shift of the photon recoil due to the index of refraction of a dilute gas of atoms has been observed. The recoil frequency was determined with a two-pulse light grating interferometer using near-resonant laser light. The results show that the recoil momentum of atoms caused by the absorption of a photon is $n \hbar k$, where $n$ is the index of refraction of the gas and $k$ is the vacuum wavevector of the photon. This systematic effect must be accounted for in high-precision atom interferometry with light gratings.

PACS numbers: 03.75.Dg,39.20.+q,42.50.Ct
\end{abstract}

The momentum of a photon in a dispersive medium is of conceptional and practical importance [1, 2, 3, 4, 5, 6]. When a photon enters a medium with index of refraction $n$, the electromagnetic momentum changes from $\hbar k$ to $\hbar k / n$, where $\mathrm{k}$ is the vacuum wavevector of the photon. Momentum conservation requires that the medium has now a mechanical momentum $(1-1 / n) \hbar k$ which for $n>1$, is parallel to the propagation of the light. The particles in the medium are accelerated by the leading edge of the light pulse and decelerated by the trailing edge. As a result, no motion is left in the medium after the pulse has passed. An absorbing surface without reflection would therefore receive a momentum of $\hbar k$ per incident photon. In contrast, a reflecting surface will recoil with a momentum of $2 n \hbar k$ per photon. In this case, the standing wave formed by the incident and reflected light pulse transfers momentum to the medium which remains even after the light pulse has left. This modification of the recoil momentum has so far only been observed for light being reflected from a mirror immersed in a liquid [7, 8].

Recently, there have been discussions about what happens to an atom when it absorbs a photon within the medium. If one assumes that after absorbing the photon, no motion is left in the medium, then the recoil momentum should be $\hbar k$ [9]. The same conclusion is reached when one assumes a very dilute, dispersive medium with the absorbing atom localized in the vacuum space between the particles of the medium [10]. However, the correct answer is that the atom will recoil with a momentum of $n \hbar k$, which requires particles in the medium to receive a backward momentum (for $n>1$ ) due to the interaction of the oscillating dipole moments of the particles in the dispersive medium and the absorbing atom. So both for reflection and absorption by an atom, a photon in a dispersive medium behaves as if it has a momentum of $n \hbar k$.

In this paper, we study the recoil momentum of a photon in a dilute gas and show that its value is $n \hbar k$. This has important consequences for atom interferometers using optical waves to manipulate atoms by the transfer of recoil momentum. High

\footnotetext{
*Present address: JILA, Boulder, CO 80309.
}

precision measurements of the photon recoil are used to determine the fine structure constant $\alpha[11,12,13,14,15,16]$. Further improvements in the accuracy of photon recoil measurements, combined with the value of $\alpha$ derived from the (g-2) measurements for the electron and positron [17, 18, 19], would provide a fundamental test of QED. The accuracy of the best photon recoil measurements are limited by the uncertainty in the correction to the photon recoil due to the index of refraction. At low atomic densities, where atom interferometers usually operate, the index of refraction effect is relatively small. Here we operate an atom interferometer with BoseEinstein condensates, which have much higher density than laser cooled atomic clouds, and observe how the index of refraction modifies the atomic recoil frequency $\hbar \omega_{\text {rec }}=\frac{p^{2}}{2 m}$, where $p$ and $m$ are the atomic recoil momentum and mass respectively.

The essential idea of our experiment is to measure the recoil frequency interferometrically using a two-pulse Ramsey interferometer. The two pulses are optical standing waves separated by a delay time $\tau$ (Fig. 11. The first pulse diffracts the atoms in an ${ }^{87} \mathrm{Rb}$ condensate into discrete momentum states. During the delay time $\tau$ the phase of each momentum state evolves at a different rate according to its recoil energy. The second pulse recombines the atoms with the initial condensate. The recombined components have differing phases leading to interference fringes that oscillate at the two-photon recoil frequency. By measuring the resulting frequency as a function of the standing wave detuning from the atomic resonance, we found a distinctive dispersive shape for $\omega_{\text {rec }}$ that fit the recoil momentum as $n \hbar k$, where $n$ is the index of refraction of the gas.

The experiment was performed using an elongated ${ }^{87} \mathrm{Rb}$ Bose-Einstein condensate created in a cloverleaf-type IoffePritchard magnetic trap using the procedure described in Ref.[20]. The condensate, containing $1.5 \times 10^{6}$ atoms, was produced in the $\left|5^{2} S_{1 / 2}, F=1, m_{F}=-1\right\rangle$ state, and had a Thomas-Fermi radius of 8 (90) $\mu \mathrm{m}$ in the radial (axial) direction, and the magnetic trap had a radial (axial) trap frequency of $81(7) \mathrm{Hz}$.

The BEC was illuminated from the side with an optical standing wave created by a retro-reflected, $\pi$-polarized laser 


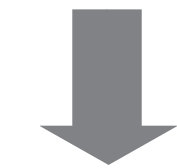

$\overrightarrow{\mathrm{B}}$
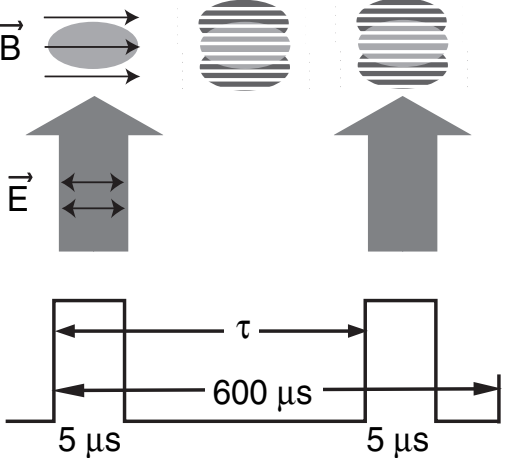

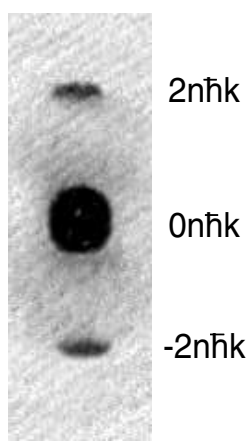

Release from Trap Image in TOF
FIG. 1: Kapitza-Dirac interferometer. The first pulse outcoupled a small fraction of atoms into the $| \pm 2 n \hbar k\rangle$ momentum states. The outcoupled atoms moved within the initial condensate. After a variable delay $\tau$ a second pulse was applied and outcoupled atoms that interfered with those outcoupled by the first pulse. The laser beam was applied perpendicular to the long axis of the condensate; the polarization, $\vec{E}$, was parallel to it and to the applied magnetic field bias, $\vec{B}$. $600 \mu s$ after the first pulse was applied the atoms were released from the magnetic trap and imaged after $38 \mathrm{~ms}$ of ballistic expansion. The field of view is $0.5 \mathrm{~mm} \times 1.5 \mathrm{~mm}$

beam. The polarization of the beam was optimized by suppressing Rayleigh superradiance [20]. The laser was detuned from the $5^{2} S_{1 / 2}, F=1 \rightarrow 5^{2} P_{3 / 2}, F=1$ transition at $\lambda=780 \mathrm{~nm}$, and had a linewidth $\gamma$ much smaller than $\Gamma$, the natural linewidth of the transition. The intensity of the $5 \mu \mathrm{s}$ long pulse was set to outcouple $\approx 5 \%$ of the atoms i nto each of the $| \pm 2 n \hbar k\rangle$ momentum states with no appreciable population in higher momentum states. This ensured that the density of the original condensate was nearly constant throughout the measurement. After a variable time $\tau$, a second identical pulse was applied. The time between the first pulse and the shutoff of the magnetic trap was fixed at $600 \mu \mathrm{s}$, which was less than a quarter of the radial trap period. The momentum distribution of the condensate was imaged after $38 \mathrm{~ms}$ of ballistic expansion, long enough for the momentum states to be resolved. The images were obtained using resonant absorption imaging after first optically pumping the atoms to the $5^{2} S_{1 / 2}, F=2$ state. To compensate for spontaneous light scattering from the standing wave, the density of the condensate (and associated mean field shift) was determined by applying a single 5 $\mu s$ pulse to the condensate, and then immediately releasing it from the magnetic trap. The number of atoms in the condensate was determined by integrating the optical density of the absorption image. The optical density was calibrated by fitting the Thomas-Fermi radius of unperturbed condensates in time-of-flight [21].

The recoil frequency was found by fitting the oscillations (a)
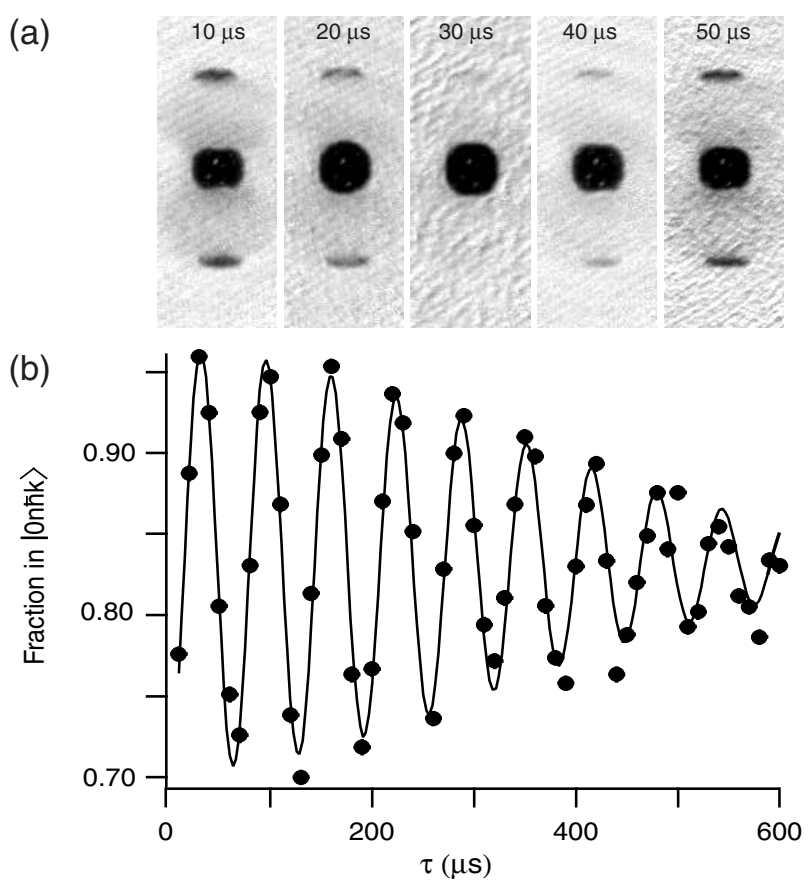

FIG. 2: Interference fringes oscillating at the recoil frequency. (a) Absorption images for $\tau=10-50 \mu \mathrm{s}$. The detuning was $\Delta / 2 \pi=$ $+520 \mathrm{MHz}$ relative to the $\mathrm{F}=1 \rightarrow \mathrm{F}^{\prime}=1$ transition. The field of view is $0.5 \mathrm{~mm} \times 1.5 \mathrm{~mm}$ (b) Fraction of atoms in the $|0 \hbar k\rangle$ momentum state as a function of $\tau$. The fringes were fit using Eq. 1. The fitted frequency was $\omega=2 \pi \times 156268(39) \mathrm{Hz}$ and the decay constant $\tau_{c}=461(25) \mu \mathrm{s}$. The signal was normalized using the total atom number in all momentum states.

in the fraction of atoms in the $|0 n \hbar k\rangle$ momentum state for $\tau$ ranging from $10-590 \mu s$ (Fig. 2). The interference fringes were fit to a cosine function with a gaussian envelope:

$$
A \exp \left(-\frac{\tau^{2}}{\tau_{c}^{2}}\right) \cos (4 \omega \tau+\phi)+C
$$

The observation of up to ten oscillations provided a precise value of the recoil frequency. The origin of the damping time $\tau_{c}$ and of the offset $C$ will be discussed later.

Figure 3 shows our measured values for $\omega / 2 \pi$ as a function of the detuning, $\Delta / 2 \pi$, relative to the $\mathrm{F}=1 \rightarrow \mathrm{F}^{\prime}=1$ transition. The measured values for the recoil frequency clearly follow the dispersive shape of the index of refraction, and they are in good agreement with the hypothesis that the recoil momentum is proportional to the index of refraction. The variation in $\omega / 2 \pi$ as a function of the detuning was $2 \mathrm{kHz}$ across the resonance, much larger than the statistical error on the frequency fits of less than $100 \mathrm{~Hz}$. This conclusively shows that the momentum transferred to the atom when a photon is absorbed is $n \hbar k$.

We now discuss in more detail how the atoms interact with optical standing waves. For the short duration of the applied pulses $(5 \mu s)$ we can assume that the atoms do not move during the pulse and ignore the kinetic energy of the atoms (Raman-Nath approximation). The interaction can then be de- 


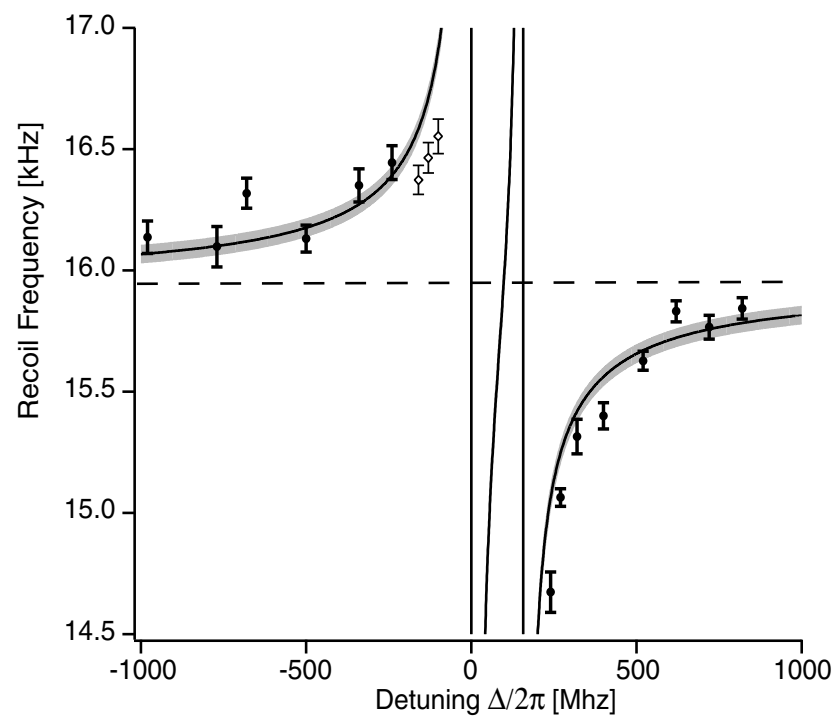

FIG. 3: Recoil frequency as a function of detuning $\Delta / 2 \pi$ showing the dispersive effect of the index of refraction. The average density of the condensate for the solid points was $1.14(4) \times 10^{14} \mathrm{~cm}^{-3}$, giving rise to a mean-field shift of $880 \mathrm{~Hz}$. The shaded area gives the expected recoil frequency including the uncertainty in the density. The dashed line is at $\omega=4 n^{2} \omega_{r e c}+\rho U / \hbar$, the expected value without index of refraction effects. The data shown as open diamonds had increased spontaneous light scattering due to $\sigma^{ \pm}$light contamination in the laser beam. The increased light scattering led to a lower initial density in the condensate, thus leading to a smaller mean-field shift and index of refraction. The $\sigma^{ \pm}$contamination allowed $\Delta m_{F}= \pm 1$ transitions, thus for small detunings the proximity to the $|1,-1\rangle \rightarrow$ $\left|0^{\prime}, 0\right\rangle$ transition located at $\Delta / 2 \pi=-72 \mathrm{MHz}$ resulted in higher spontaneous scattering rates. The open points have been scaled upward to correct for this lower density.

scribed by the application of the AC Stark potential due to the standing wave

$$
V(z)=\frac{\hbar \omega_{R}^{2}}{\Delta} \sin ^{2}(n k z),
$$

where $\Delta$ is the detuning between the optical frequency and the atomic transition, and $\omega_{R}$ is the Rabi frequency. This equation is valid for large detuning, $\Delta^{2} \gg \Gamma^{2} / 4$, where $\Gamma$ is the natural linewidth of the transition. The short pulse limit, describing Kapitza-Dirac scattering, is valid for short interaction times $t_{p}$, where $t_{p} \ll 1 / \omega_{r e c} \approx 40 \mu \mathrm{s}$. The first pulse outcouples a fraction of atoms into the momentum states $| \pm 2 \ell n \hbar k\rangle$, where the population in the $\ell^{\text {th }}$ momentum state is given by $P_{\ell}=$ $J_{\ell}^{2}(\theta)$ [22, 23], where for a square pulse, $\theta=\frac{\omega_{R}^{2} t_{p}}{2 \Delta}$, and $J_{\ell}$ is the $\ell^{t h}$-order Bessel Function of the first kind. For $\theta<$ 1 a negligible fraction of atoms is diffracted into states with $\ell>1$, and we can restrict our discussion to the $| \pm 2 n \hbar k\rangle$ momentum states. For our experimental parameters $\theta=0.45$. During the delay time $\tau$ the phase of the $| \pm 2 n \hbar k\rangle$ states evolves at a faster rate than the $|0 n \hbar k\rangle$ state due to the recoil energy, $E_{r e c}=4 n^{2} \hbar \omega_{r e c}$, hence the wavefunction will evolve as

$$
|\psi(\tau)\rangle=\left|\psi_{o}\right\rangle\left(J_{1}(\theta)| \pm 2 n \hbar k\rangle e^{-i 4 n^{2} \omega_{r e c} \tau}+J_{0}(\theta)|0 n \hbar k\rangle\right) .
$$

At $t=\tau$ a second pulse is applied that partially recombines the momentum states. After applying the two pulses, the probability of finding the atoms in the the $|0 n \hbar k\rangle$ momentum state, $\rho_{0}=\left|\left\langle\psi\left(\tau+t_{p}\right) \mid 0 n \hbar k\right\rangle\right|^{2}$ is given by

$$
\rho_{0}=J_{0}^{4}(\theta)+4\left[J_{0}^{2}(\theta) J_{1}^{2}(\theta)+J_{1}^{4}(\theta)\right] \cos \left(4 n^{2} \omega_{r e c} \tau\right) .
$$

As a function of $\tau$ the density of the zero momentum peak oscillates at $4 n^{2} \omega_{\text {rec }}$.

So far, we have ignored the motion of the atoms during the delay time $\tau$. However the amplitudes of the recombined components will only interfere where they spatially overlap. After the first pulse, the atoms in the $| \pm 2 n \hbar k\rangle$ momentum states move within the initial condensate with the recoil velocity ( $\sim 12 \mu \mathrm{m} / \mathrm{ms})$. As the overlap between the recoiling atoms and those at rest decreases, the interference fringes decay. The overlap integral for this decay can be approximated as a Gaussian with time constant, $\tau_{c} \approx 0.75 R_{T F} / v_{r e c}$, where $R_{T F}$ is the Thomas-Fermi radius of the condensate, and $v_{r e c}$ is the recoil velocity [24].

The index of refraction for the condensate is derived from its macroscopic polarization $P$. For a two level system,

$$
P=\chi \epsilon_{o} E=i \rho \frac{\mu^{2}}{\hbar} \frac{E}{\Gamma-i \Delta}
$$

where $\chi$ is the atomic susceptibility, $\epsilon_{o}$ is the permittivity of free space, $\mu$ is the dipole matrix element, $\rho$ is the atomic density of the condensate. In this experiment the light was $\pi$-polarized and detuned by $\Delta$ from the $5^{2} S_{1 / 2}, F=1 \rightarrow 5^{2} P_{3 / 2}, F^{\prime}=1$ transition. For this polarization the selection rule is $\Delta m_{F}=0$, and there are two allowed transitions from $\left|F=1, m_{F}=-1\right\rangle \rightarrow\left|1^{\prime},-1\right\rangle$ and $|1,-1\rangle \rightarrow\left|2^{\prime},-1\right\rangle$ which are separated by $157 \mathrm{MHz}$. Including both transitions in the derivation, the index of refraction, $n=\sqrt{1+\chi}$, is given by:

$$
\begin{aligned}
& n= \\
& \sqrt{1-12 \pi \rho\left(\frac{\lambda}{2 \pi}\right)^{3}\left(\frac{5}{12} \frac{\Delta_{1}}{\Gamma} \frac{1}{1+\left(2 \frac{\Delta_{1}}{\Gamma}\right)^{2}}+\frac{1}{4} \frac{\Delta_{2}}{\Gamma} \frac{1}{1+\left(2 \frac{\Delta_{2}}{\Gamma}\right)^{2}}\right)}
\end{aligned}
$$

where $\Delta_{1}$ and $\Delta_{2}$ are the detunings relative to the $F=1 \rightarrow$ $F^{\prime}=1$ and $F^{\prime}=2$ transitions, respectively. This equation is valid in the limit $\rho \lambda^{3} \ll 1[25]$, where $\lambda=\lambda / 2 \pi$. For our experimental parameters $\rho \lambda^{3} \approx 0.2$. In addition to the index of refraction shift, the observed recoil frequency has a mean field shift [26]; the atoms in the $| \pm 2 n \hbar k\rangle$ state have twice the mean field energy of those at rest due to the exchange term in the interatomic potential. Including both the mean field shift and the index of refraction, the frequency of the observed interference fringes should be:

$$
\omega=4 n^{2} \omega_{r e c}+\frac{\rho U}{\hbar}
$$


where $\rho U=4 \pi \hbar^{2} a \rho / m$, and $a$ is the s-wave scattering length. The density $\rho=4 / 7 \rho_{o}$, where $\rho_{o}$ is the peak condensate density and the factor of $4 / 7$ is due to the inhomogeneous condensate density.

When the interference fringes were fit using Eq. (1), the average values for the amplitude $A$ and offset $C$ for all of the data points were $0.12(3)$ and $0.82(4)$, respectively. This is in reasonable agreement with the expected values of $A=0.18$ and $C=0.81$ for $\theta=0.45$. For a Thomas-Fermi radius of $8 \mu \mathrm{m}$ we would expect a decay time $\tau_{c} \approx 500 \mu \mathrm{s}$. There was an unexplained shift in the fitted value for the decay time $\tau_{c}$ between the red and blue side of the resonances, on the red side the average value was $347(20) \mu$ s and on the blue, 455(40) $\mu \mathrm{s}$.

The quadratic dependence in Eq. (7) on the index of refraction can be understood by considering the diffraction of atoms from the light grating created by the standing wave. When the first pulse is applied, the standing wave creates a grating with a periodicity $d=\lambda / 2$. Within the condensate the index of refraction modifies the grating period by $n$, since $\lambda^{\prime}=\lambda / n$. The recoil momentum of atoms that diffract off the grating will be changed by $\pm 2 \hbar k^{\prime}$, again within the condensate $k^{\prime}=n k$ and the velocity of the atoms is modified. Assuming $n>1$, when the second grating is applied the atoms have moved farther by a factor of $n$ and the grating is shorter by $n$, changing the time scale for the interference fringes by a factor of $n^{2}$.

The increase in the momentum transferred to the atoms can also be explained by considering the momentum transferred to atoms by a classical field. A derivation using the Lorentz force applied to the atoms during the absorption of a photon can be found in [6]. In a dielectric medium with $n>1$, the magnetic field and therefore the Lorentz force are not modified. However, the electric field is weaker and therefore it takes longer for the atom to perform half a Rabi cycle and be transferred to the excited state. During that longer time, the Lorentz force imparts a momentum to the atom which is larger than $\hbar k$.

For Kapitza-Dirac scattering, atoms are diffracted symmetrically into the into $| \pm 2 \ell n \hbar k\rangle$ momentum states, so momentum is clearly conserved. However for processes such as Bragg scattering, where the atoms are scattered in only one direction the index of refraction has an additional effect. Assuming a $\pi / 2$ pulse with counter-propagating beams, where half the atoms are diffracted, for $n>1$ the recoil momentum is a factor of $n$ higher than the vacuum momentum. For momentum to be conserved, the remaining atoms must recoil backwards with momentum $p=2(n-1) \ell \hbar k$. For small fractional outcoupling the effect is negligible, since the extra momentum is distributed among the remaining condensate. However if a large fraction of the condensate is outcoupled and $\ell$ is large, this effect could potentially be resolved in ballistic expansion.

We have discussed here the dispersive effect on the photon momentum near a one-photon resonance. An analogous effect occurs near two-photon resonances. In this case, the atomic polarizability is determined in third-order perturbation theory, and the resulting index of refraction has a sharp, narrow dis- persive feature near the two-photon resonance [27]. In recent experiments at Stanford [13], such two-photon effects have been the leading source of uncertainty in high-precision determinations of atomic recoil frequencies and the fine-structure constant $\alpha$.

In conclusion we have measured a systematic shift in the photon recoil frequency due to the index of refraction of the condensate. This is the first direct observation of the atomic recoil momentum in dispersive media. For high atomic densities, this shift can have a significant effect on atom interferometers, and is of particular importance for precision measurements of $h / m$ and $\alpha$ with cold atoms [13, 16].

The authors thank A. Schirotzek, S. Chu, S. Harris and H. Haus for insightful discussions, and M. Kellogg for experimental assistance. This work was supported by NSF and ARO.

† URL: http://cua.mit.edu/ketterle_group/

[1] H. Minkowski, Nachr. Ges. Wiss. Göttingen p. 53 (1908).

[2] H. Minkowski, Math. Ann 68, 472 (1910).

[3] M. Abraham, Rend. Pal 28, 1 (1909).

[4] M. Abraham, Rend. Pal 30, 33 (1910).

[5] J. P. Gordon, Phys. Rev. A 8, 14 (1973).

[6] M. P. Haugan and F. V. Kowalski, Phys. Rev. A 25, 2102 (1982).

[7] R. V. Jones and J. C. S. Richards, Proc. R. Soc. London A221, 480 (1978).

[8] R. V. Jones and B. Leslie, Proc. R. Soc. London A360, 347 (1978).

[9] J. M. Hensley, A. Wicht, B. C. Young, and S. Chu, in Atomic Physics 17, edited by E. Arimondo, P. D. Natale, and M. Inguscio (2001).

[10] This argument has been made previously by one of the authors (W. K.).

[11] B. Taylor, Metrologia 31, 181 (1994).

[12] D. S. Weiss, B. C. Young, and S. Chu, Phys. Rev. Lett 70, 2706 (1993).

[13] A. Wicht, J. M. Hensley, E. Sarajlic, and S. Chu, Physica Scripta 102, 82 (2002).

[14] S. Gupta, K. Dieckmann, Z. Hadzibabic, and D. E. Pritchard, Phys. Rev. Lett 89, 140401 (2002).

[15] R. Battesti, P. Cladé, S. Guellati-Khèlifa, C. Schwob, B. Grémaud, F. Nez, L. Julien, and F. Biraben, Phys. Rev. Lett. 92, 253001 (2004).

[16] Y. L. Coq, J. A. Retter, S. Richard, A. Aspect, and P. Bouyer, ArXiv: cond-mat/0501520 (2005).

[17] R. S. Van Dyck, P. B. Schwinberg, and H. G. Dehmelt, Phys. Rev. Lett 59, 26 (1987).

[18] T. Kinoshita, Phys. Rev. Lett 75, 4728 (1995).

[19] V. W. Hughes and T. Kinoshita, Rev. Mod. Phys 71, S133 (1999).

[20] D. Schneble, Y. Torii, M. Boyd, E. W. Streed, D. E. Pritchard, and W. Ketterle, Science 300, 475 (2003).

[21] Y. Castin and R. Dum, Phys. Rev. Lett 77, 5315 (1996).

[22] P. Meystre, Atom Optics (Springer-Verlag, New York, 2001).

[23] S. Gupta, A. E. Leanhardt, A. D. Cronin, and D. E. Pritchard, C.R. Acad. Sci. IV-Phys. 2, 479 (2001).

[24] M. Trippenbach, Y. B. Band, M. Edwards, M. Doery, P. S. Julienne, E. W. Hagley, L. Deng, M. Kozuma, K. Helmerson, S. L. 
Rolston, et al., J. Phys. B 33, 47 (2000).

[25] O. Morice, Y. Castin, and J. Dalibard, Phys. Rev. A. 51, 3896 (1995).

[26] J. Stenger, S. Inouye, A. P. Chikkatur, D. M. Stamper-Kurn,
D. E. Pritchard, and W. Ketterle, Phys. Rev. Lett 82, 4569 (1999).

[27] A. Schirotzek, Diploma thesis, Universität Hamburg (2004). 